\title{
AN EVALUATION APPROACH FOR DETECTION OF CONTOURS WITH 4-D IMAGES: A REVIEW
}

\author{
Asmita A. Pawar ${ }^{1}$, Anita L. Devkar ${ }^{2}$, Ashwini A. Patil ${ }^{3}$ \\ ${ }^{1}$ Lecturer, IT Department, MCOE, Pune, Maharashtra, India \\ ${ }^{2}$ Lecturer, IT Department, MCOE, Pune, Maharashtra, India \\ ${ }^{3}$ Lecturer, IT Department, MCOE, Pune, Maharashtra, India
}

\begin{abstract}
Abstract This paper presents a survey of contour detection and the actual use of contour in image processing. Image processing is an enhanced area in computer science. Contour detection is the part of image processing. Contours are highly depends on quality of an image. Contour is nothing but the simple boundaries or outlines in an image. Contour detection is nearly related with image segmentation, classification and recognition of any object in an image. With help of contour detection we can achieve the high accuracy of the results. Object recognition image retrieval uses the concept of contour detection to achieve the high accuracy in the results, so it's an enhanced and popular method in image processing. Active contour model is also one of the main techniques in contour detection. Active contour is one of the successful models in image processing. This is a modified method of contour detection. It consists of evolving an image with help of boundaries. Active contour model is also called as snake. Contour detection plays an important role in recognition.
\end{abstract}

Keywords: 4D images, Contour Detection, Image Segmentation, Image Classification etc...

\section{INTRODUCTION}

Contour is the basic outline representation of any structural diagram. Contour detection is an enhanced problem in image processing. This issue is related with connected contours. Contour need to be closed curves. When contours are obtained from edges then there is necessary condition to form the contours with closed edges. This is necessary to solve the contour problem. There are so many problems with image processing such as contour, image noising and blurring in mage. So it is the necessary technique to avoid all these problems. The challenge with the solution is that the original image should not be get affected. Contours are depends on the appearance of an image. The initial stage in solving that problem is that to detect the unwanted things in mage and then try to repair them. The detection of missing parts and to solve them is essential. Different image processing critical problems like image inpainting and denoising (or enhancement) are solved without detecting the boundaries and contours. The major goal of image enhancement technique is to filter the image without blurring it. A basic idea is initially to identify the boundaries in order to store them and to smooth the image which is outside. In this critical case, contour completion is not an initial solution, as the properties of the final result is not too much comparable to the completeness of the identified boundaries, but some missing boundaries may lead to blurred boundaries.

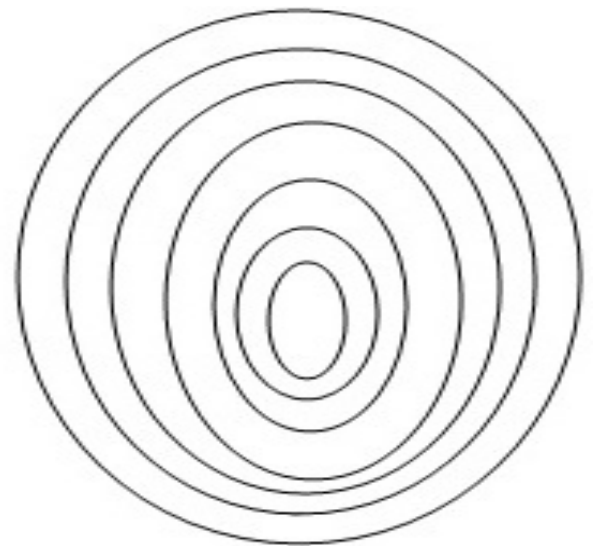

Fig. 1 Basic diagram of Contour

Contour is the basic fundamental in image processing. With help of contour we can detect the lines and with help of detection we can achieve our final result of detection of an image. Recent approaches to contour detection are at quantifying the presence of a boundary at a given image location through the local measurements. Contour is the part of any pattern in an image. Active contour model is one of the frameworks for the detection of outlines in an image. With help of contour detection any user can detects the outlines in an image and then after detection try to remove the unwanted area in an image. Contour tracing is the backbone concept of contour detection. Contour tracing is one of the preprocessing scenarios. Initially contours are detected and then the properties of the contours are observed by the user [11]. After observing the features, that features are highly used for classification in an image. The resultant of the contour detection is the final outcome from the features of an image. 
For so many image processing critical problems (segmentation, classification, image inpainting), the detection of connected contours can tremendously simplify the resolution and highly improve the quality of the final results.

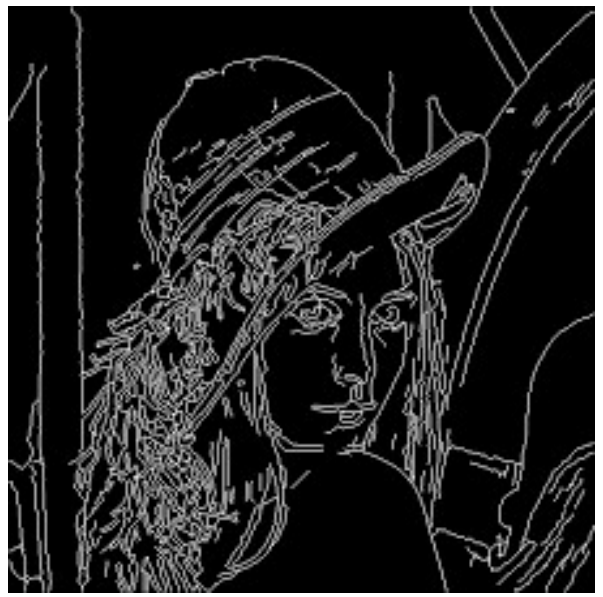

Fig. 2 Contour Detection in an Image

\section{METHODOLOGY USED}

\subsection{Edge Detection}

Edge detection is the method for detection which having goal to identify points in an image which the effects of image are changes. With help of edge detection we can detect the boundaries in the image and then we detect the final result. Any image is consisting of pixels and the combination of pixel forms the edge [1]. The first step of the approach is to finding out the number of pixels in the given image and then finds out the connected curve in the particular image. This is a nontrivial problem by image itself, because there are so many effects with different types of images. There are so many effects with the images such as brightness, luminance etc. So it is necessary to maintain all the effects after forming changes in the image.

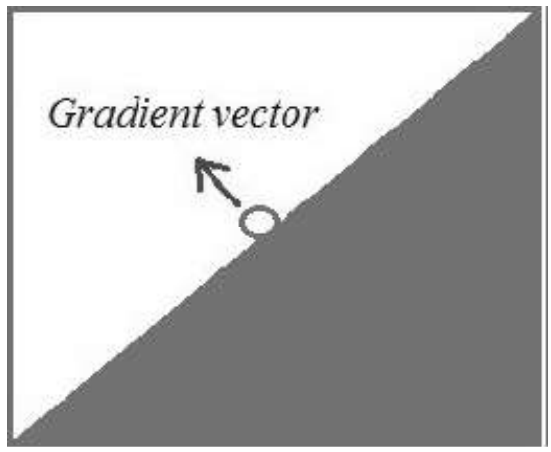

Fig. 3 Edge Detection in Facial Image

\subsection{CONTOUR DETECTION}

Contour detection is a critical issue in image processing. In classification we use the technique to split original image into the number of parts. This serious occurrence is highly related to the detection of the contours which are connected together. It is sometimes easy to detect boundaries using local image analysis techniques, but the detection of the continuous contours is more difficult and needs a major analysis of the image. In this typical case, contour detection is not an initial approach, as the characteristics of the images are not too much related with the completeness of the image which is identified[5]. Most of the image having some missing parts and blurring in it, so it is necessary to solve that problem with help of contours. The contours in the image are highly helpful for detection. With help of contour detection we can also increase the recognition rate of the image [2].

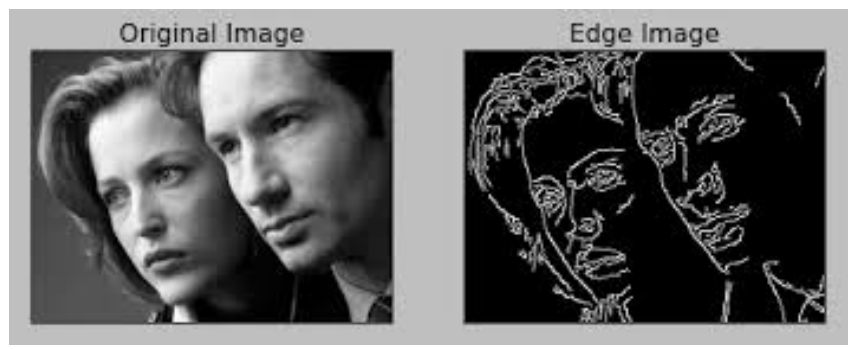

Fig. 4 Basic diagram of Contour

\subsection{Image Segmentation}

The main goal of image segmentation is to form the clusters of number of pixels into image regions. Image segmentation is an important technique in image processing. A segmentation technique is used for object recognition, occlusion detection and removal and estimation. Image segmentation is highly used to extract the number of segments from the tested image. For the input we primarily considered the effects in the image [3][4]. There is need to maintain all the effects after applying algorithm on it. Segmentations in the simple level images can provide a useful data about the surfaces in the given image. The number of pixels is followed by the image. Then form the clusters of that image. After forming the cluster we apply the filtering on it [9]. The quality of the segmentation is highly depends on the image. Clustering is an important method for image segmentation. With help of clustering we can categorize the properties of clusters into separate groups. There are different techniques of clustering such as mean segmentation and mean iterations [6]

An accurate segmentation is highly used for accurate detection in the given image.

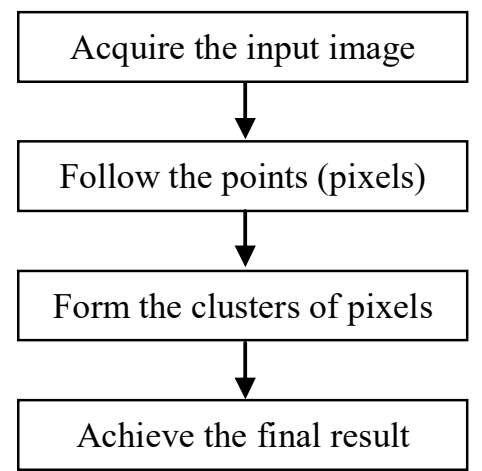

Fig. 5 Flow of Image Segmentation 


\section{RESEACH DIRECTIONS}

\subsection{Study On Different Techniques For Detection}

Face recognition is an enhanced task of recognizing a sample using digital face images. There are so many techniques for detection such as face recognition, face segmentation, contour detection and classification. An image recognition system is typically depends on recognition based on similar features. We can recognize faces with help of similar features. Initial step is to form the cluster of nearest matching features and then on the basis of cluster we can detect the accurate results [12].

\subsection{Study The Detection On 4-D Images}

Initial stage of the algorithm is to obtain the .obj file from the camera of 4 Dimensions. After reading that obj, the mesh structure is formed using MATLAB [4]. An accurate extraction of the different features of the face are highly depends on the detection of the tip point of the nose. It is necessary to identify the clear and accurate features of the face image [8].

\section{CONCLUSIONS}

In this paper, we have described the methods for detection of an image and also discussed the contour detection technique. The contour is highly useful for detection in the image. Image processing domain having lots of techniques for detection but he contour detection is an useful method because it highly focuses on the boundaries in the images. By combining edge detection and contour detection we can get the better results for detection. With help of contour we can formulate the number of boundaries in the given input image. Contour detection is an enhanced method for detection of the images.

\section{ACKNOWLEDGEMENT}

I would like to thank my dear friends for their sincere discussions and valuable feedback. I would also like to thank my guide. I express my warm gratitude to them for their unconditional support whenever and where ever it was required.

\section{REFERENCES}

[1]. Bo Wang, Kun Zhang, Liang Shi, Huihui Zhong, “ An Edge Detection Algorithm of Moving Object Based on Background Modeling and Active Contour Model“, International Conference on Systems Engineering and Modeling, pp. 340-346, 2013.

[2]. Manjeet Kaur and Sukhpreet Kaur, "Role of Active Contour in Image Processing," International Journal of Science and Research(IJSR), vol. 3, Issue 5, May 2014.

[3]. Kar, S., Hiremath, S., Joshi, D.G., Chadda, V.K., Bajpai, A.: A Multi-Algorithmic Face Recognition System. In: International Conference on Advanced Computing and Communications, pp. 321-326 (2006)

[4]. Draper, B.A., Baek, K., Bartlett, M.S., Ross BeveRidge, J.: Recognizing Face with PCA and ICA.
Computer Vision and Image Understanding 91, 115137 (2003)

[5]. Ch.Sri Veera Sagar, K.Rama Devi, “A New Approach Of Edge Detection In SAR Images Using RegionBased Active Contours", International Journal of Research in Engineering and Technology, vol.2, September 2013.

[6]. Kalpana Saini, M.L. Dewal, and Manojkumar Rohit, "Level set based on new Signed Pressure Force Function for Echocardiographic image segmentation", International Journal of Innovation and Applied Studies, vol.3 No. 2, pp. 560-569, June 2013.

[7]. http://en.wikipedia.org/wiki/Image_segmentation.

[8]. Sara Memar, Karen Jin, Boubakeur Boufama, "Object Detection Using Active Contour Model with Depth Clue", Image Analysis and Recognition, Lecture Notes in Computer Science, Vol. 7950, 2013.

[9]. Yunyun Yang, Shenzhen Grad, "Split Bregman method for minimization of modified Vese-Chan model for fast image segmentation", ISPA-2013.

[10].Phung, S.L., Bouzerdoum, A., Chai, D.: Skin Segmentation Using Color And Edge Information. In: International Conference on Signal Processing and Its Applications, vol. 1, pp. 525-528 (July 2003).

[11]. http://www.di3d.com

[12].Wang, Z., Huangfu, F.K., Wan, J.W.: Human Face Feature Extraction Using Deformable Templates. Journal of Computer Aided Design and Computer Graphics of China 12(5), 333-336 (2000).

\section{BIOGRAPHIES}

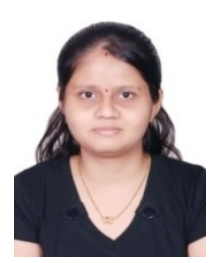

Asmita A. Pawar is a lecturer in the Information Technology Department, Modern College of Engineering, Pune ,India. She received Master of Engineering (ME) degree from North Maharashtra University, Jalgaon, India. Her research interests are Image Processing and Technologies.

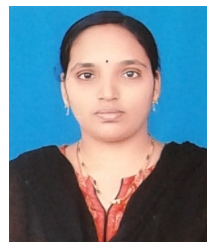

Anita L. Devkar is a lecturer in the Information Technology Department, Modern College of Engineering, Pune, India. She received Master of Technology (M Tech) degree from COE, Pune. Her research interests are Information Retrieval, Data Mining and Cloud Computing.

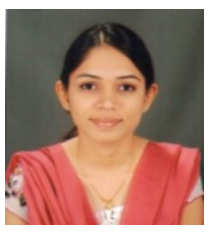

Ashwini A. Patil is a professor in the Information Technology Department, Modern College of Engineering, Pune, India. She received Master of Engineering (ME) degree from, Pune University. Her research interests are Software Engineering and Data Mining. 